\title{
Optogenetisch bestätigt: Schöne Erinnerungen vertreiben den Trübsinn
}

\author{
Ein paar Gedanken an ein schönes Erlebnis - und schon wird die Stimmung \\ besser. Werden positive Erinnerungen aktiviert, können sie Depressionen fast \\ völlig auslöschen - das klappt sogar im Tierversuch.
}

D ie Kinderbuchgeschichte des Grafikers und Schriftstellers Leo Lionni von Frederick der Maus beschreibt in einfachen Wort die Kraft positiver Erinnerungen: Die Feldmaus Frederick sitzt den ganzen Sommer scheinbar untätig herum, während die anderen Mäuse Vorräte für den Winter sammeln. Auf die Frage, warum er nicht mithelfe, sagt er nur, er sammle doch auch, und zwar Sonnenstrahlen, die Lieder der Vögel, Wörter und Farben. Schließlich kommt der lange Winter, die Vorräte werden weniger und die Stimmung sinkt auf einen Tiefpunkt. Dann wird Frederick nach seinen Vorräten gefragt. Er erzählt nun von den Blumen, den Farben des Frühlings, singt die Lieder der Vögel, und den Mäusen wird warm ums Herz: Sie vergessen den Winter und merken gar nicht, wie schnell der Frühling kommt. Lionni beschreibt mit seiner Geschichte einen simplen antidepressiven Mechanismus: Wer es schafft, positive Erinnerungen $\mathrm{zu}$ reaktivieren, kann trübsinnigen Gedanken die Stirn bieten.

\section{LED im Kopf}

Professor Volker Arolt vom Universitätsklinikum Münster erinnerte anhand der Geschichte von Frederick, welch mächtiges Werkzeug Therapeuten hier zur Verfügung steht. Inzwischen können Forscher anhand ausgeklügelter Tierexperimente sogar belegen, wie gut Gedächtnisengramme Depressionen bekämpfen und wie dieser Mechanismus funktio- niert. Gelungen ist das - wie könnte es anders sein - mithilfe von Mäusen.

Die moderne Variante von Frederick wird von japanischen Optogenetikern um den Nobelpreisträger Professor Susumu Tonegawa erzählt [Ramirez $\mathrm{S}$ et al. Nature 2015; 522: 335 -9]. Sie implantierten den Tieren einen Schalter gezielt in Neurone, die bei bestimmten positiven Erinnerungen aktiv werden. Der Schalter besteht aus einem Kanalrhodopsin, einem Ionenkanalprotein aus Blaualgen. Bei bestimmten Wellenlängen öffnet sich der Ionenkanal und aktiviert das Neuron. Mit einer LED im Schädel können die Forscher die damit bestückten Neurone in Echtzeit an- und ausschalten. Das Rhodopsin wird über einen viralen Vektor in das Gehirn verfrachtet, ein spezielles System sorgt dafür, dass es nur zu bestimmten Zeitpunkten in die gerade aktiven Neurone eingebaut wird.

Nun suchten die Forscher einen positiven Reiz für die Mäuse. Sie entschieden sich für die Kopulation. Die ist zwar wenig kinderbuchgeeignet, gilt aber als eines der stärksten positiven Ereignisse im Mäuseleben. Sexuell ausgehungerte Männchen durften sich einige Zeit bei weiblichen Artgenossen austoben, genau in dieser Zeit wurde der Einbau des Rhodopsin-Schalters aktiviert. Er sollte sich also vorwiegend in den Neuronen finden, die bei sexueller Aktivität beteiligt sind. Zudem wurde der Einbau räumlich auf den Gyrus dentatus begrenzt - letzt-

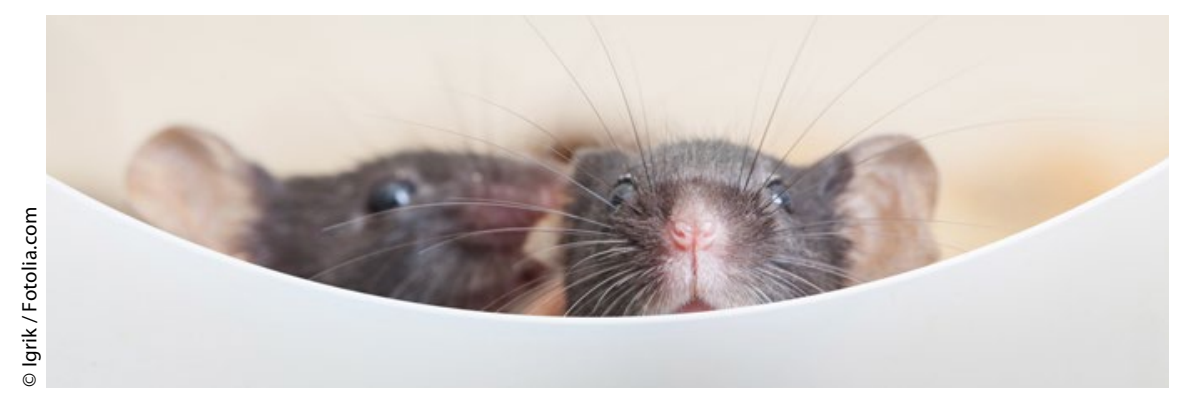

lich sollten diejenigen Nervenzellen markiert werden, die an der Engrammierung beteiligt sind.

\section{Mehrfache Reaktivierung löscht die Depression}

Im nächsten Schritt setzten die Forscher die Mäuse unter chronischen Stress, indem sie sie täglich zwei bis drei Stunden fixierten. Nach zehn Tagen zeigten sie ein deutliches depressions- und angstähnliches Verhalten. So wirkten sie im Vergleich zu ungestressten Tieren etwa apathisch, wenn man sie am Schwanz hochzog. Schalteten die Forscher nun drei Minuten lang das LED-Licht im Schädel ein, verschwanden die Depressionssymptome völlig. Nach mehrfachem An- und Ausschalten blieb der antidepressive Effekt sogar dauerhaft bestehen. Die Mäuse waren dann ebenso „glücklich" wie diejenigen ohne traumatische Erlebnisse, sie zeigten sogar weniger Depressionssymptome als Tiere, die nach dem Immobilisationsstress zu den Weibchen durften. Der tatsächliche Sex nach dem Trauma wirkte offenbar weniger antidepressiv wie der zuvor positiv engrammierte. Umgekehrt konnten die Forscher bei Mäusen mit der LED im Kopf auch negative Emotionen aktivieren, und zwar wenn der Rhodopsin-Einbau während der Stressphasen stattfand. Die Wissenschaftler vermuten, dass es bei wiederholter Reaktivierung der Erinnerung $\mathrm{zu}$ neuroplastischen Veränderungen kommt, offenbar wird dabei die Neurogenese im Hippocampus angekurbelt.

Die Resultate seien natürlich nur mit Vorsicht auf Menschen zu übertragen, so Arolt. Doch würden ähnliche Effekte bei Psychotherapien beobachtet, die auf eine Aktivierung positiver Erinnerungen setzen, etwa bei Strategien zum „mood repair". Solche werden nicht zuletzt auch bei demenzkranken Menschen verwendet: Hier können Musik und eine vertraute Umgebung positive Erinnerungen triggern und unruhige oder aggressive Menschen wieder etwas beruhigen.

Thomas Müller

Professor Volker Arolt: Depression.

Psychiatrie Update, 11.- 12.3.2016, Wiesbaden 